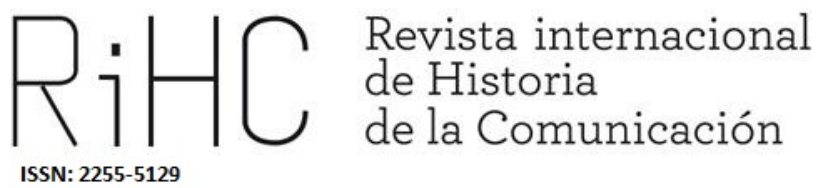

\title{
LA PRENSA CATÓLICA DE FRANCO: ¡ZAS! UN MODELO DE REVISTA INFANTIL (1945)
}

\section{Franco's catholic press: Zas! A model of children's magazine (1945)}

DOI: http://dx.doi.org/10.12795/RiHC.2018.i10.13

Recibido: 03/05/2018

Aceptado: $28 / 05 / 2018$

Publicado: 25/06/2018

Lucia Ballesteros-Aguayo (iD https://orcid.org/0000-0003-1191-4070

Universidad de Sevilla, Iballesteros@us.es

Resumen: Este trabajo aborda el análisis descriptivo de carácter histórico de la primera revista infantil de Acción Católica durante la posguerra española: iZas! Auspiciada por la doctrina del nacionalcatolicismo y promovida por las organizaciones religiosas, la nueva política comunicativa adoptada por el régimen franquista a partir de 1945 propició el surgimiento de una prensa catequística que tenía como objetivo contribuir a la acción pastoral de la Iglesia Católica. Destinada a los aspirantes de Acción Católica, iZas! se convirtió en sus ocho meses de edición en el prototipo de publicación infantil, inaugurando así una tradición periodística que cristalizó en el surgimiento de cabeceras confesionales y en una mayor presencia de la Iglesia Católica en la edición periódica Palabras clave: prensa, infancia, Franco, propaganda, Segunda Guerra Mundial, Iglesia 
Abstract: This research addresses the descriptive analysis of historical character of the first children's magazine published by Catholic Action during the Spanish post-war period: iZas! Created in the light of the political doctrine of the nacionalcatolicismo and in the heart of the religious organizations, the new media policy adopted by the Franco regime as of 1945, encouraged the emergence of a catechetical press focused on the pastoral activity of the Catholic Church. iZas! was intended for the aspirants of the Catholic Action and became the children's model magazine in just eight months, thus beginning a press tradition that strengthened the emergence of confessional newspapers and the role of the Catholic Church in the newspaper and magazine publishers

Keywords: children's magazine, Spanish post-war period, Franco, propaganda, Catholic Church

\section{Introducción}

La prensa como vehículo de transmisión cultural promueve la preservación de unos derechos fundamentales necesarios para el desarrollo de la persona $y$, al mismo tiempo, impulsa la plena integración del ciudadano en la sociedad. Además, la utilización de los soportes periodísticos favorece y estimula la intercomunicación social y contribuye a promover la acción cultural-informativa como principio consustancial al propio sistema democrático.

La educación infantil alcanza una singularidad exclusiva en su difusión a través de los medios de comunicación, especialmente a través de los canales de la Iglesia Católica debido a su carácter confesional. La transmisión de modelos de conducta y la difusión de un conjunto de valores sociales, políticos y culturales contribuyen de manera significativa a la configuración de una determinada concepción de la niñez y, por tanto, a la reconstrucción de toda una época1.

La utilización propagandística de las revistas infantiles según el esquema ideológico de posguerra respondía a una estrategia política concreta y singular2. Por consiguiente,

\footnotetext{
1 MONTERO GARCIA, F. (2005): «Las publicaciones periódicas de Acción Católica durante el Franquismo», en: RUIZ SÁNCHEZ, J.L. (Ed.), Catolicismo y comunicación en la historia contemporánea. Sevilla: Universidad de Sevilla, p. 34, señala la importancia de la prensa como fuente de conocimiento de la realidad histórica y social de España -en concreto, este autor se refiere a las revistas editadas por Acción Católica en los años precedentes a la guerra civil española- porque "a falta de otras fuentes primarias, estas publicaciones han servido para la reconstrucción de la historia de las Mujeres, y los Jóvenes de AC en los años 20 y $30 "$.

${ }^{2}$ Ya lo apuntaba MARTíNEZ CUESTA, F. J. y ALFONSO SÁNCHEZ, J.M. (2013): "Tardes de enseñanza y parroquia. El adoctrinamiento de las niñas en la España franquista a través de las revistas "Bazar" y "Tin Tan" (1947-1957)». El Futuro del Pasado. Revista electrónica de historia, no 4, p. 233, citando a su vez a CIRICI, A. (1977): La estética del franquismo. Barcelona: Gustavo Gili, y refiriéndose a las revistas
} 
resulta relevante el análisis de la transmisión de un determinado discurso periodístico que conformó la realidad de varias generaciones.

Dicho estudio se hace imprescindible en situaciones como la actual en la que, a pesar de la literatura científica al respecto y del creciente interés por la prensa editada durante este periodo3, aún existe un número limitado de propuestas que aborden el análisis de las principales cabeceras de posguerra, cuestión que ya ha sido apuntada por los especialistas en la materia 4.

Al respecto, son múltiples los autores que reivindican la necesidad de abordar una revisión científica de los periódicos de posguerra, en especial acerca del periódico que se convirtió en paradigma de la prensa falangista, Flechas y Pelayos, y de las iniciativas que surgieron, sobre todo a partir de 1945, vinculadas a los organismos eclesiásticos como entidades impulsoras de una nueva fórmula en la edición infantil.

No obstante, existen aportaciones recientes que constituyen un precedente significativo en el estado de la cuestión. Algunas de ellas son: Blasco Herranz, I. (2005) que aborda el estudio de la Sección Femenina y de Acción Católica5, Corderot, D. (2000) que estudia ampliamente el proceso de adoctrinamiento político ideológico de la infancia durante la guerra civil española 6 o los trabajos de Martínez Cuesta, F. J. y Alfonso Sánchez, J.M. (2013) en torno al análisis de publicaciones infantiles de posguerra como Bazar y Tin Tan?.

Se hace, pues, necesario la realización de trabajos que aborden el análisis de la producción periodística de carácter infantil de posguerra para alcanzar una mejor

editadas por Acción Católica durante los años posteriores a la guerra civil española: "Lo cierto es que todas estas publicaciones infantiles en cierta medida, dependiendo de su difusión, representaron en la posguerra el factor cultural y la presencia artística más considerable de la vida del Estado Español pasando a ser objetivamente la representación más importante de la cultura de hechos".

${ }^{3}$ Algunas de las aportaciones más significativas son: Viñetas de posguerra. Los cómics como fuente para el estudio de la historia de Oscar Gual Boronat o La prensa infantil en España: desde el siglo XVIII hasta nuestros días de Mercedes Chivelet. Junto a ello se multiplican las tesis doctorales, seminarios, coloquios, artículos, ponencias y jornadas que reflexionan sobre el fenómeno periodístico en torno a la infancia durante el periodo de posguerra.

${ }^{4}$ Véase, por ejemplo, LARA, A. (2002): «Los tebeos del franquismo», en: Viviane, A. (Ed.), Historietas, comics y tebeos españoles. Toulouse: Presses Universitaires du Mirail, p. 49: "Está por hacer una investigación minuciosa de Flechas y Pelayos, sin prejuicios, examinando cuidadosamente todo lo que se publicó en sus páginas, para salvar lo salvable -que fue abundante y de una gran creatividad, a pesar de las censuras y del miedo a meter la pata en cualquier momento, dada la fragilidad de las normas y orientaciones-, y rechazar las aportaciones estúpidas, dañinas y sin sentido [...]".

${ }^{5}$ BLASCO HERRANZ, I. (2005): «Sección Femenina y Acción Católica. La movilización de las mujeres durante el franquismo». Gerónimo de Uztariz, no 21, pp. 55-56.

${ }^{6}$ CORDEROT, D. (2013): «La Guerre d'Espagne racontée aux enfants et par les enfants dans les revues enfantines nationalistes (1936-1939)», en: HERVOUËT, C. et al., Enfants en temps de guerre et littératures de jeunesse (20-21e siècles). Clermont-Ferrand: Presses universitaires Blaise Pascal.

${ }^{7}$ MARTÍNEZ CUESTA, F. J. y ALFONSO SÁNCHEZ, J.M., op.cit. 
comprensión no sólo del fenómeno comunicativo durante este periodo, sino también de la dimensión ideológica y política de estos acontecimientos mediáticos que se convierten a su vez en fuentes de información de la realidad histórica de nuestro país.

\section{Objetivos y Metodología}

Los objetivos específicos que representan las metas a alcanzar a través de esta investigación son:

1) Identificar las peculiaridades periodísticas que presenta la revista infantil iZas! de Acción Católica, así como los aspectos formales más significativos de su evolución.

2) Valorar la trascendencia que ejerce iZas! tanto en el entramado editorial de la posguerra, como en las publicaciones posteriores editadas por organismos religiosos.

3) Analizar la influencia de iZas! en el establecimiento de un determinado modelo de prensa religiosa a partir de 1945.

4) Identificar los hechos en el ámbito comunicativo más significativos que impulsaron una nueva política editorial por parte del régimen de Franco en relación a las revistas infantiles religiosas.

5) Revindicar el papel esencial que desempeñan estas publicaciones en el proceso de transmisión de un determinado universo icónico infantil.

El marco metodológico de esta investigación es preferentemente de carácter histórico, que se fundamenta en la comprensión del fenómeno periodístico a través de la información que proporciona su historia.

La metodología de carácter histórico se explica por las interacciones entre el contexto comunicativo y el ámbito histórico-político, lo cual hace que sea indispensable acudir a las fuentes históricas para definir una perspectiva científica del fenómeno estudiado.

Gracias a la metodología histórica se ha acometido el estudio de los materiales hemerográficos correspondientes a la revista iZas! Para ello se ha realizado a priori una búsqueda documental exhaustiva en los fondos tanto privados como públicos. Así, por ejemplo, se han consultado los archivos de la Hemeroteca Municipal de Madrid, así como se ha realizado un registro de ejemplares a través de las webs y portales de coleccionistas y asociaciones disponibles en internet.

El objetivo final era recabar el número total de ejemplares de iZas! durante su escaso, pero significativo periodo de edición. De manera que a pesar de que la revista se 
publicó desde marzo de 1945 hasta octubre de ese mismo año, actuó como un hito importante en la prensa confesional del régimen, puesto que inauguró un periodo a partir del cual proliferarían las publicaciones de Acción Católica con una marcada misión apostólica y catequística.

Después de un proceso de búsqueda hemerográfica, el número total de ejemplares analizados en esta investigación que estaban disponibles en los fondos consultados asciende a cinco, desde el inicio de la edición de la revista en marzo de 1945 con el número 1 hasta su finalización el día 28 de octubre de ese mismo año con el número $16^{8}$. Dicho corpus se identifica, pues, con: el no 1, marzo de 1945, el no 9, 1 de julio de 1945, el no 10, 15 de julio de 1945, el no 15, 15 de octubre de 1945 y el no 16, 28 de octubre de 1945.

Precisamente, la fecha de su publicación resulta esencial en la trayectoria de iZas! Así, y siguiendo una secuencia cronológica de la política comunicativa de los primeros años del régimen franquista que se inicia con la aprobación en 1938 de la Ley de Serrano Suñer, podemos establecer dos etapas bien diferenciadas dentro de la prensa infantil adscrita a los órganos estatales. Por una parte, la etapa $a z u l^{9}$ que se desarrolla como consecuencia de la creación de la red de periódicos integrados dentro del Movimiento Nacional y en la que la prensa del partido único aglutinó casi en solitario cualquier manifestación comunicativa. Y una segunda etapa a partir de 1945 cuando se produce el impulso de la prensa católica editada por congregaciones religiosas e instituciones pías como consecuencia, entre otros factores, del cambio de política internacional del régimen franquista a causa del final de la II Guerra Mundial y otros factores internos de España como la renovación del gobierno franquista o el cambio de la política informativa.

Junto a este criterio de orden temporal existen otros de carácter periodístico que facilitan el análisis de iZas! como, por ejemplo, la entidad que se encarga de su edición, el ámbito de difusión, la duración de su edición, el diseño formal, el carácter de los temas y de las ilustraciones, etc. En este punto, la consulta de fuentes hemerográficas ha sido fundamental en el análisis experimental, ya que permite definir de manera exhaustiva los rasgos definitivos del soporte periodístico. De manera que, junto al método histórico y al criterio temporal, el siguiente elemento metodológico es el uso de la técnica descriptiva, que ha permitido la descripción y el análisis crítico de la trayectoria histórica y la situación presente del objeto de análisis. Por tanto, la revisión documental representa una cuestión esencial porque ayuda a

\footnotetext{
${ }^{8}$ Ver Figura I: Portada inaugural de iZas!, Año I, no 1, marzo de 1945, p. 1.

${ }^{9}$ El término azul alude a la simbología de la Falange y a su prevalencia en todos los aspectos de la dictadura durante la primera mitad de la década de los 40 cuando el partido único controlaba no sólo los medios de propaganda, sino también los instrumentos de socialización. Es el tiempo de la creación de las instituciones de encuadramiento civil como el Sindicato Vertical y el Frente de Juventudes, así como la conformación de una red del funcionariado en torno a la Falange.
} 
situar los antecedentes y el estado actual de los presupuestos teóricos y metodológicos.

Efectivamente, el uso de la perspectiva descriptiva es fundamental para comprender el fenómeno estudiado porque facilita la ordenación, caracterización y clasificación de los materiales hemerográficos del modo más concreto posible. No en vano, la investigación descriptiva resulta esencial en determinados campos científicos como es el de las Ciencias Sociales, y especialmente en nuestro estudio, en el que el nivel de aprehensión de la realidad es escaso debido a la limitación de trabajos y al grado de condicionamiento en el que se encuentran las conclusiones alcanzadas por los mismos. Por ello se hace necesaria la definición exhaustiva del problema como fase previa al nivel de la explicación, ya que ésta puede resultar errónea si no va precedida de una correcta descripción y clasificación.

Por tanto, la influencia de la perspectiva descriptiva es consustancial a esta investigación, puesto que con ella nos proponemos detallar el comportamiento que adquiere el fenómeno periodístico a partir de sus principales rasgos definitorios con el objetivo de proceder a determinar su evolución, su desarrollo y su influencia en el periodismo posterior.

En definitiva, una metodología múltiple y complementaria que posibilita obtener conclusiones válidas y fiables acerca del fenómeno periodístico en su conjunto, principalmente desde una perspectiva comunicativa, pero también histórica y política. De manera que las manifestaciones periodísticas de posguerra pueden abordarse como expresión de la actividad comunicativa como testimonio histórico y manifestación política en el estudio de las estructuras de poder y el devenir histórico, pero también como agentes de socialización y educación.

\section{Por una prensa infantil catequística y pedagógica (1938-1945)}

Para profundizar en el estudio de los principales periódicos infantiles de posguerra es necesario comprender cuál es el entramado editorial en torno al Movimiento que comenzó a configurarse en el bando nacional durante la guerra civil. Para ello cabe destacar las revistas infantiles de principios de los años 40 que estaban inspiradas en el espíritu falangista de José Antonio Primo de Rivera -que ejerció gran influencia sobre el carácter ideológico del nuevo régimen-, la Comunión Tradicionalista Carlista -que dotó al franquismo de una amplia tradición histórica- y la Iglesia Católica -que proveyó al gobierno de una escala de valores morales, influenció en la educación de 
los españoles y desempeñó una importante labor de legitimación de la actuación del gobierno autoritario manifiesta tanto durante el conflicto como a lo largo de la dilatada dictadura ${ }^{10}$.

Especial atención merece la orientación confesional de la prensa franquista porque representa un rasgo definitorio del caso español que lo distingue del resto de regímenes fascistas de mediados del siglo $\mathrm{XX}$, por ejemplo, del fascismo de Mussolini ${ }^{11}$.

La naturaleza católica del Estado se estableció como una de las peculiaridades del franquismo por dos motivos fundamentales. En primer lugar, porque la doctrina de la Iglesia posibilitaba el corpus argumentativo necesario para justificar las causas y consecuencias de la guerra civil, interpretada a partir de ahora como una cruzada contra los infieles ${ }^{12}$. Y en segundo lugar, porque dicha interpretación otorgaba validez y legitimidad al poder autoritario ${ }^{13}$.

La influencia de la doctrina cristiana en el franquismo respondió a una cuestión no sólo estratégica, sino también simbólica enmarcada en un entramado de intereses. Dicha relación quedó refrendada por los acuerdos aprobados entre el Estado y el Vaticano. Así, en 1938 se produjo el reconocimiento oficial del gobierno de Franco por el Papa y, posteriormente, en septiembre de 1953 tuvo lugar la firma del Concordato con la

\footnotetext{
${ }^{10}$ HERRERO SUÁREZ, H. (2007): Un yugo para los Flechas. Educación no formal y adoctrinamiento infantil en Flechas y Pelayos. Lleida: Milenio, p. 105: “Así, resultaba imprescindible, a no ser que se pretendiera perpetuar el control, la represión y la violencia -algo, por otra parte, realmente difícil en largas duraciones-, que la sociedad asumiera de forma generalizada la legitimidad del sistema, estuviera persuadida de la conveniencia de su mantenimiento y aceptara, ya activa, ya pasivamente, los presupuestos ideológicos que estructurasen y articulasen los regímenes en cuestión".
}

${ }^{11}$ MURIALDI, P. (1986): La Stampa del regime fascista. Roma-Bari: Laterza, p. 69: “Il 13 maggio, concludendo a Montecitorio il dibattito sulla ratifica degli accordi col Vaticano, Mussolini lancia un ammonimento ai fogli di indole ecclesiastica e, senza nominarle, all'Azione cattolica e alle altre organizzazione laiche della Chiesa. "ll regime è vigilante -dice il duce- e nulla gli sfugge. nessuno creda che l'ultimo fogliucolo che esce nell'ultima parrocchia non sia, ad un certo punto, conosciuto da Mussolini. non permetteremo resurrezioni di partiti e di organizzazioni che abbiamo per sempre distrutti»".

12 Uno de los argumentos que la Iglesia aportó al nuevo régimen con el fin de dotar de coherencia al nuevo sistema político y de manera especial para justificar el estallido del conflicto civil y del golpe de Estado, fue la necesidad de iniciar una cruzada contra el infiel, y una campaña de erradicación del comunismo y del bolchevismo, que eran considerados por la propaganda como los mayores enemigos de la "nación española". Este soporte de justificación constituyó uno de los fundamentos del mensaje proselitista y doctrinario transmitido a través de los medios de comunicación, siendo una constante tanto en la prensa falangista como en la católica, las referencias a la "santa cruzada", así como al origen divino de Franco como líder no sólo político y militar, sino también religioso.

13 HERRERO SUÁREZ, H., op. cit., pp. 105-106: "Para ello, los diferentes sistemas políticos han considerado necesaria su legitimación ante la población, para lo que han tenido que dotarse de un discurso interpretativo, tanto cognitivo como normativo, que recogiese sus ideas y sus creencias, y que ofreciese unas determinadas escalas de valores a las que estarían indisociablemente unidos unos modelos actitudinales y comportamentales". 
Santa Sede. Estos avances reafirmaban de manera pública y ante las potencias internacionales la legitimidad de la dictadura y la nueva apariencia externa del país basada en la democracia orgánica y en el nacionalcatolicismo. Para ello Franco se fundamentó en el patrocinio de la Iglesia y en el origen confesional del Estado ${ }^{14}$.

Esta justificación se fundamentaba en la tradición católica de España recuperando el concepto de la unión religiosa en torno a la Patria que promovieron los Reyes Católicos $^{15}$. La Iglesia brindó a Franco las explicaciones teológicas de naturaleza patriótica y divina necesarias para convertirlo en garante y valedor de dicha tradición, y en el mayor defensor de la integridad y unión del país ante la amenaza del comunismo y el bolchevismo. Este hecho explica la presencia de símbolos de la época de la Reconquista en la propaganda franquista como, por ejemplo, el uso continuado del yugo y las flechas, así como múltiples tradiciones y signos que el franquismo incorporó del ideario católico ${ }^{16}$. Por tanto, la Iglesia facilitó el argumento ético y moral a la causa de Franco para justificar la guerra, y más tarde actuó como elemento legitimador del continuismo de la tradición española.

Pero la implicación en la constitución del nuevo régimen por parte del poder religioso no se limitó solamente a su participación como componente esencial del discurso ideológico ni a su influencia en el rumbo de las primeras medidas adoptadas por la nueva política, sino que se estableció como uno de los ejes clave sobre los que se fundó el Estado y en torno al cual se instauraron los organismos institucionales.

De manera que la presencia del mensaje de apostolado y la misión catequística se expandieron alrededor de todo el territorio nacional a través de organizaciones religiosas como la ACNP. Conviene señalar al respecto el dominio que ejerció la ACNP tanto en la composición de los gobiernos franquistas -especialmente a partir de 1945 con la instauración de la democracia orgánica- como en la orientación de la política

\footnotetext{
${ }^{14}$ Hacia este respecto se dirigen las reflexiones de CARR, R. (1999): España, de la Restauración a la democracia, 1875-1980. Barcelona: Ariel, 6ạ ed., p. 200: "La conquista real de la Iglesia consistió en recobrar el sistema educativo. Se purgó a los maestros republicanos, aquellos que se quedaron estuvieron sujetos a cursos intensivos de reeducación en el pensamiento católico".

${ }^{15}$ CARR, R., op. cit., p. 200: “Por su apariencia exterior y por el lenguaje político, el nuevo Estado de Franco parecía una recreación del reino de los Reyes Católicos con algún ribete de la Italia fascista. La despreciable retórica de los primeros momentos del régimen, aunque incomprensible para una mente racional, se difundió en un intento por 'renovar' la tradición con el fin de reconciliar el siglo XVI con la reacción autoritaria moderna contra 'la democracia inorgánica de los partidos'".

${ }^{16}$ GÓMEZ MOMPART, J. L. (2002): «Ecosistema comunicativo franquista y construcción simbólica y mental de España», en: GARCÍA GALINDO, J. A.; GUTIÉRREZ LOZANO, J. F.; y SÁNCHEZ ALARCÓN, I. (Eds.), (2002), La comunicación social durante el franquismo. Málaga: CEDMA, p. 604: "La construcción simbólica como paso previo a la consiguiente construcción social y la subsiguiente construcción mental. De ahí la necesidad de una sistemática educación sentimental como manto que arropa a la educación histórica, de ahí la siembra de las semillas simbólicas para que después germinen los hitos históricos por irracionales que resulten".
} 
comunicativa o el papel esencial que desarrollaron entidades como Acción Católica en las instituciones de educación de la sociedad española ${ }^{17}$.

Así, se estableció la exención de la censura previa para las publicaciones editadas por Acción Católica -de la que ya disfrutaban los periódicos de la Falange desde 1942 por una Orden del 24 de febrero-, lo que promovió la aparición de la prensa católica financiada por las instituciones religiosas, y el surgimiento de cabeceras de periodicidad no declarada.

Esta tendencia se amplió y extendió durante la posguerra con la instrumentalización de los periódicos infantiles por parte del Estado ${ }^{18}$ que cumplieron con dos objetivos fundamentales: legitimar el poder instaurado por la fuerza e instruir a las futuras generaciones en los principios del nacionalsindicalismo.

\section{Héroes cristianos y moral católica: iZas!, la primera revista infantil de los aspirantes de Acción Católica}

Precisamente a partir de 1945 se incrementó la actuación de organizaciones como Acción Católica ${ }^{19}$ y, en definitiva, la presencia de la Iglesia Católica en la edición periódica. Esto es así debido a los cambios experimentados tanto en el contexto político de país con la incorporación de miembros de la ACNP al gobierno franquista, como en el ámbito internacional. Todo lo cual promovió que la prensa católica

\footnotetext{
${ }^{17}$ Con el franquismo la mayoría de los organismos de enseñanza se integraron bajo el poder de la Iglesia y a ellos se les atribuía la responsabilidad de educar a los españoles en el ideario franquista. Especial significancia merece el carácter que adquiere dicha formación sobre los más pequeños. Si bien es cierto que desde el comienzo de la dictadura la catolicidad del Estado ocupó un lugar preferente, no fue hasta la aprobación de la Ley de Ordenación Universitaria el 29 de julio de 1943, y especialmente a partir de 1945 con la nueva política de gobierno, cuando multiplicó su dominio sobre todos los niveles educativos, incluyendo la formación universitaria. Dicha ley recoge en su artículo tercero el carácter manifiestamente católico de la educación superior franquista: "La Universidad, inspirándose en el sentido católico, consubstancial a la tradición universitaria española, acomodará sus enseñanzas a las del dogma y de la moral católica y a las normas del Derecho canónico vigente". BOE, núm. 212, 31-071943.

${ }^{18} \mathrm{El}$ franquismo se propuso desarrollar en torno a la prensa un concreto proyecto formativo sobre la retórica del providencialismo y la concepción ideológica de la historia, basándose para ello en la repetición de tópicos de brevísimo fundamento ontológico más que en una doctrina sistemática y consistente. Cfr. HERRERO SUÁREZ, H., op. cit., p. 87.

${ }^{19}$ Para un estudio pormenorizado sobre la naturaleza y origen de Acción Católica, consultar MONTERO GARCIA, F., op. cit.
} 
comenzara a adquirir mayor relevancia con la multiplicación de soportes de clara orientación parroquial y militante destinadas a los más pequeños ${ }^{20}$.

El primer testimonio fue iZas! que inauguró un periodo en la prensa infantil caracterizado por la influencia de Acción Católica sobre las publicaciones periódicas ${ }^{21}$. Por tanto, y a pesar de que su breve periodo de existencia -el primer número se corresponde con la edición de marzo de 1945 y el último, con el no 16, de 28 de octubre de ese mismo año-, iZas! ejerció un papel significativo en el cambio del paradigma comunicativo, que irá evolucionando progresivamente desde una prensa adscrita al control del partido único hasta el dominio cada vez más presente de la Iglesia Católica.

Editada como "Revista Nacional de la Sección de los Aspirantes de Acción Católica", algunos autores han denominado a este tipo de publicaciones como "tebeos o revistas infantiles", entre los que diferenciaban los tebeos comerciales, los tebeos confesionales y los que llaman de indoctrinación política "cuya finalidad era convencer al público infantil sobre determinadas formas de comportamiento colectivo" ${ }^{22}$.

El soporte nació con un objetivo principal: contribuir y ampliar la misión de apostolado promovida por las órdenes y las congregaciones religiosas. Su edición periódica facilitaba la presentación de contenidos amenos a través del relato ficcional, $y$, por tanto, se convertía en un instrumento idóneo para transmitir el mensaje de la Iglesia sirviéndose del carácter lúdico de la prensa.

Así, pues, el discurso periodístico se convirtió en un instrumento esencial para adoctrinar en un determinado modelo de educación religiosa y así garantizar la formación católica de las futuras generaciones.

\footnotetext{
${ }^{20}$ Tal y como apunta Ibídem, p. 33, refiriéndose a los objetivos que debían de cumplir los soportes periódicos de Acción Católica en España: "Las publicaciones son una necesidad para la buena marcha coordinada de las organizaciones (en ellas se difunden las orientaciones y los métodos...), pero sobre todo para el cumplimiento de sus objetivos misioneros, que implican la difusión de unos determinados valores aplicados a los respectivos ambientes...".

${ }^{21}$ Sólo precedida por Signo que actuó como Semanario Nacional de la Juventud de Acción Católica en España. Para más información, consúltese MONTERO GARCIA, F., op. cit., p. 34: "Pocos meses antes del comienzo de la guerra civil nació el periódico de la Juventud de AC, Signo, que se publicó ininterrumpidamente durante la guerra civil hasta su desaparición en 1967, coincidiendo con la crisis de la ACE. Su trayectoria refleja la de la propia organización juvenil desde el clima triunfalista y nacionalcatólico de los 40 al despegue crítico de los 60".
}

${ }^{22}$ MARTÍNEZ CUESTA, F. J. y ALFONSO SÁNCHEZ, J.M., op. cit., p.233. 


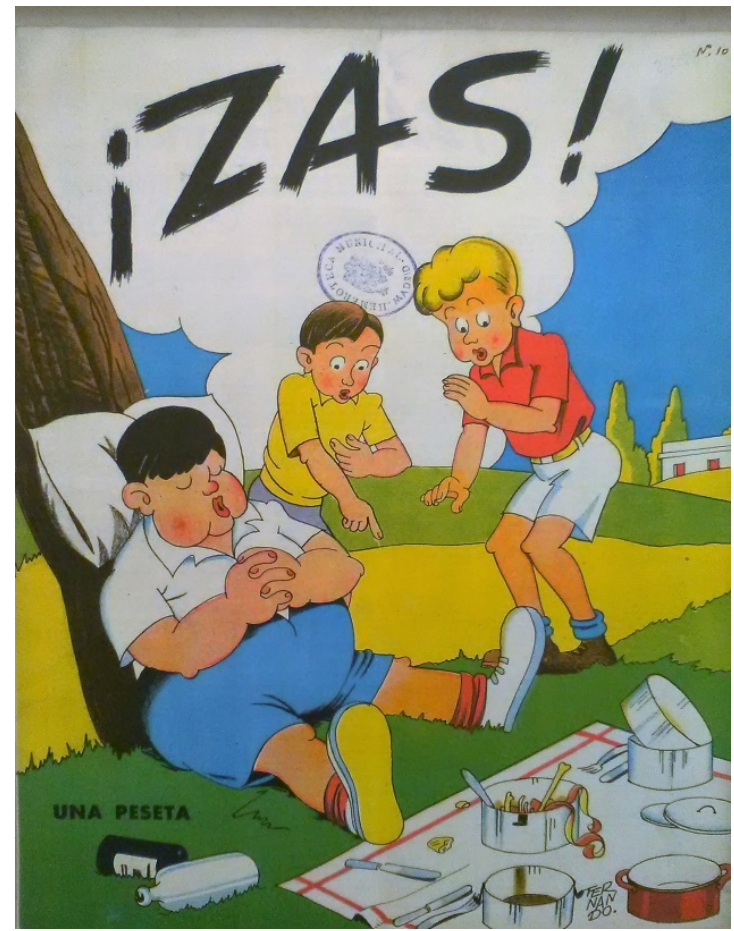

Figura 1. Portada de iZas!, Año I, no 10, 15 de julio de 1945, p. 1

\subsection{Descripción formal: el universo icónico de los más pequeños}

Dichos cambios encuentran su justificación en la descripción formal de los materiales hemerográficos que ejemplifican a su vez la evolución que experimentó el soporte en su edición.

Con unas dimensiones de $27 \times 21 \mathrm{~cm}$., la publicación comienza su edición con una portada en la que destaca el uso del color, y la inclusión de un dibujo a gran escala que ocupa toda la página -normalmente firmado por Fernando-. Tan sólo incorpora el precio -1 peseta-, y el título de la revista ${ }^{23}$.

Ya en la parte interior, en la segunda página, aparecen diferentes secciones entre las que destacan "iZas! en el mundo", y "Cómo viviremos mañana", destinadas a la publicación de noticias y hechos significativos relacionados con la ACNP. Y no es hasta la página tercera cuando aparecen los datos de la revista. Así, la página tres incorpora la cabecera en la parte superior y, dentro de ésta, el "Año", que no corresponde al año natural, sino que se identifica con la edición de la revista, es decir, la publicación establece una cronología específica a partir de su primer número.

\footnotetext{
${ }^{23}$ Ver Figura 1. Portada de iZas!, Año I, no 10, 15 de julio de 1945, p. 1 (Anexo I).
} 
Junto al "Año" se localizan otros datos como el número, y la siguiente aclaración: "Publicación quincenal en domingos alternos con censura eclesiástica".

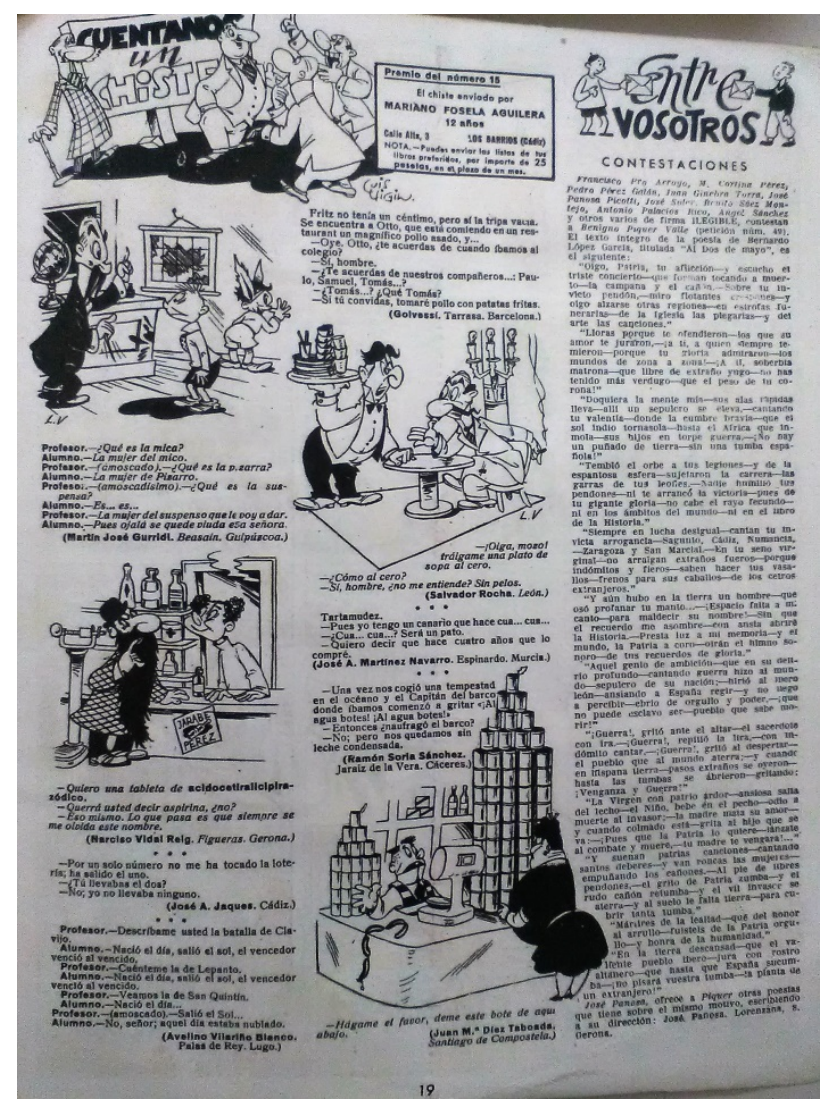

Figura 2. Sección "Entre vosotras”, iZas!, Año I, no 16, 28 de octubre de 1945, p. 19

En la parte central se sitúa el título de la publicación y la fecha -que en el caso de la primera edición se correspondía con la "segunda quincena de marzo" porque la revista comienza siendo quincenal y, luego, más tarde, adopta una periodicidad semanal-, y a continuación se incluye un recuadro horizontal que separa la cabecera del resto de la página en el que se inserta el subtítulo "Revista Nacional de la Sección de los Aspirantes de la Acción Católica".

Por último, en la parte superior derecha de la cabecera se incorporan los datos referentes a: "Redacción y administración: Conde de Xiquena, 5, Madrid", "Se publica en domingos alternos, con censura eclesiástica" y "Suscripción anual: 25 pesetas; semestral: 12,50 pesetas".

Esta presentación varía a lo largo del tiempo, y ya en el no 26 de agosto de 1945 se produce el primer cambio con la modificación del formato de la cabecera de la primera página impar. En esta edición lo primero que aparece es "Revista Nacional de la Sección de los Aspirantes de la Acción Católica", le sigue a la izquierda "Redacción administración: Conde de Xiquena, 5, Madrid", y "Suscripción anual: 25 pesetas; 
semestral: 12,50 pesetas", en la parte central se especifica el "Año", la fecha, el no y "Se publica en domingos alternos, con censura eclesiástica", y en la parte superior derecha los precios de suscripción.

En cuanto a la periodicidad, la revista presenta dos etapas: una que se extiende desde el no 1 de marzo de 1945 hasta el no 11 de 5 de agosto de 1945 en la que la difusión es quincenal, y otra etapa a partir de esta fecha cuando la revista comienza a editarse semanalmente. Con una media de 20 páginas, el texto del interior solía estar dividido en dos columnas, y la impresión se realizaba en los talleres Offset de San Sebastián.

En cuanto a la distribución de las secciones, la portada se caracteriza por la incorporación de un dibujo que ocupa toda la página.

En la página segunda aparece bien un cómic titulado "Episodios históricos", o bien un apartado destinado a curiosidades con el nombre de "iZas! alrededor del mundo".

La página tercera está protagonizada por un cuento de J. Ministral "Camino del instituto", "Una tarde en el pinar" o "El primer pitillo" y un relato lúdico ilustrado que suele ser "El libro de la selva. Los hermanos Mowgli" por Rudyard Kipling y que incluye la siguiente advertencia "con autorización de la Editorial Gustavo Gil, S.A.". Y más tarde -en concreto a partir del no 15 de 15 de octubre de 1945- por un cómic lúdico de Moro.

La siguiente sección ocupa una doble página y está a color. Se destina a la presentación de un cómic o a una historieta con viñetas -suele ser habitual la serie "Extraño caso de la Gallina Taciturna"-. Más tarde incorpora una página de curiosidades.

Los apartados siguientes publican un cuento educativo, habitualmente firmado por José Luis Peñuela, como puede ser el caso de "La nobleza de Guillermo" publicado en el no 13 de 16 de septiembre de 1945.

A continuación, se presenta una sección reservada para el cómic seriado en "El pequeño profesor Pin, y su ayudante Freddy" que destaca por su uso del color además de otras historietas con viñetas, como "La pantera negra" o "El Buque Fantasma".

En la página trece o catorce destaca un espacio destinado a "Filatelia", que estaba firmado por José Ma Francés, y otro a "Ajedrez" por Juan M. Fuentes junto a datos curiosos, noticias y resultados deportivos en "Fútbol".

En torno a la página quince o dieciséis aparece la sección objeto de nuestro análisis. Es una de las pocas páginas que se imprimen a color, y en ella se publica un cuento seriado de carácter doctrinario bajo el título de "Aspirantes". Más tarde se incorpora un espacio destinado a la historieta cómica en "Los rayos". 
Las páginas finales se reservan para la publicación de "Concursos" y "Crucigramas", un cuento con viñetas -como "El oso encantado" o "En busca de la rosa azul"-, y a la correspondencia con los lectores en "Entre vosotros" 24.

Por último, destaca la inclusión de un apartado dedicado al cómic, o secciones que promueven la participación del lector, tales como "Cuéntanos un chiste".

La contraportada suele dedicarse a "Tu Biblioteca. Concurso-encuesta" o a un cómic tal es el caso de "Hazañas de Teseo"- y a la publicidad con la inserción de un anuncio que ocupa toda la página que podía referirse al lanzamiento de una nueva publicación, es el caso de "Cumbres", en el número 2 de la segunda quincena de marzo.

En suma, y a modo de síntesis, los apartados de la revista que presentan cierta regularidad se corresponden con el relato lúdico ilustrado "El libro de la selva. Los hermanos Mowgli" por Rudyard Kipling, el cómic "Episodios históricos", las historietas con viñetas de la serie "Extraño caso de la Gallina Taciturna" y la correspondiente a "La pantera negra", el cómic seriado "El pequeño profesor Pin, y su ayudante Freddy" (a color), el espacio reservado a "Filatelia" y "Ajedrez", y "Fútbol" destinado a curiosidades, la sección propagandística "Aspirantes" (a color), los "Concursos" y "Crucigramas", el apartado reservado a la correspondencia con los lectores en "Entre vosotros" y, por último, la sección "Cuéntanos un chiste".

En definitiva, todo un alarde de propuestas de lectura para los más pequeños en los que se integraban secciones destinadas al ocio y al recreo con propuestas propiamente ideológicas cuya función era explícitamente formativa e instructiva.

\section{Conclusiones}

A partir de las premisas teóricas anteriormente apuntadas podemos concluir que el análisis de los símbolos de la comunicación representa una oportunidad de estudio y una propuesta de análisis necesaria no sólo porque actúan como fuente histórica de importante valor, sino porque además contribuyen a describir las propiedades del discurso periodístico y a señalar los parámetros científicos necesarios para su estudio. Esta tarea posee especial relevancia si tenemos en cuenta las singularidades del contexto político de la posguerra marcado por las exigencias ideológicas, el control de la censura y el carácter doctrinario de la prensa.

Todo ello explica que en este trabajo se haya adoptado una perspectiva multidisciplinar de carácter histórico que ha permitido descubrir nuevos aspectos del

\footnotetext{
${ }^{24}$ Ver Figura 3. Sección “Entre vosotras”, iZas!, Año I, no 16, 28 de octubre de 1945, p. 19 (Anexo I).
} 
paradigma ideológico del franquismo -que adquiere unas peculiaridades específicas en su transmisión a través de la prensa-, y la adecuación de éste a las características específicas del fenómeno periodístico, a saber, una difusión periódica y regular, la importancia del elemento visual, el carácter lúdico de la lectura, etc.

Precisamente una de las prioridades del régimen fue adoctrinar a los futuros ciudadanos en el ideario franquista eligiendo como instrumento formativo la prensa, de ahí su carácter pedagógico especialmente presente en los soportes de carácter religioso. Por tanto, el discurso periodístico se sustentaba en su contribución al proceso de instrucción de la infancia y la juventud.

En este contexto hay que situar la edición iZas!, que inaugura un nuevo modelo de prensa infantil inspirada en los principios de la moral cristiana y editada por organismos eclesiásticos como Acción Católica. Desde que comenzara con el primer número en marzo de 1945 hasta el último en octubre de ese mismo año, el soporte se convirtió en el paradigma de las nuevas producciones periódicas de índole religioso destinadas a la infancia, iniciando así una nueva etapa en la producción periodística que evolucionó como consecuencia del propio desarrollo del medio periodístico y de la industria editorial aún incipiente.

Una prensa de carácter pedagógico cuya finalidad esencial era contribuir a la misión catequística de la Iglesia Católica, al tiempo que formaba a los futuros ciudadanos. De manera que, a partir de su publicación, muchas fueron las producciones que seguirían su estilo, su presentación formal y su concepción narrativa (inspirándose ésta a su vez en soportes anteriores también pertenecientes a Acción Católica como por ejemplo el Semanario Nacional de la Juventud de Acción Católica, Signo).

En iZas! están presentes los principios que tenían que orientar la conducta del aspirante de Acción Católica, de ahí su carácter instructivo y pedagógico, pero también está presente el uso del color con fines estéticos, el arte de la ilustración, el universo icónico del niño y la diversión y el aspecto lúdico propio de la infancia. Todo lo cual explica que surgieran más tarde cabeceras homólogas, tal es el caso de Alba de Juventud (1946), Volad (1948) o Trampolín (1949).

Por tanto, una propuesta periodística de gran diversidad que apostaba tanto por la lectura de recreo, con apartados dedicados exclusivamente al ocio como pueden ser "Crucigramas" o "Fútbol", como por el relato narrativo o el cómic de aventuras, es el caso de "El libro de la selva" o "El pequeño profesor Pin, y su ayudante Freddy", junto con secciones reservadas a la propaganda de Acción Católica, sin olvidar el uso del humor, la paradoja y la risa como elementos esenciales de la interacción periodística con la infancia. 


\section{Referencias bibliográficas y fuentes}

\section{Fuentes hemerográficas}

Hemeroteca Municipal de Madrid

iZas! [en papel]. Madrid: Sección de los Aspirantes de la Acción Católica, marzo de 1945-28 octubre de 1945. (n.1, marzo de 1945- n.16, 28 de octubre de 1945) [consulta: 05-04-2016]. [Signatura: 901/3].

\section{Bibliografía}

ALARY, V. (Ed.), (2002): Historietas, comics y tebeos españoles. Toulouse: Presses Universitaires du Mirail.

ALTARRIBA, A. (2001): La España del tebeo. Madrid: Espasa Calpe.

ÁlVAREZ, J.T. et al. (1989): Historia de los medios de comunicación en España. Periodismo, imagen y publicidad (1900-1990). Barcelona: Ariel.

AUBERT, P. y DESVOIS, J. M. (Eds.), (1996): Presse et pouvoir en Espagne, 1868-1975. Bordeaux-Madrid: Maison des Pays Ibériques-Casa de Velázquez.

BALLESTEROS AGUAYO, L. (enero, 2014): «Volad. Una propuesta de intercomunicación con la infancia». Revista ISL de la Asociación Española de Comprensión Lectora (AECL), no 1, pp. 57-63.

BIESCAS, J. A. y TUÑóN DE LARA, M. (1982): «España bajo la dictadura franquista (1939-1975)», en: TUÑÓN DE LARA, M. (Dir.), Historia de España, tomo X. Barcelona: Labor, 2 a ed.

CARR, R. (1999): España, de la Restauración a la democracia, 1875-1980. Barcelona: Ariel, 6a ed.

CASASÚS, J. M. (1998): Ideología y análisis de los medios de comunicación. Barcelona: CIMS, 4aㅡ ed.

CENDÁN, F. (1986): Medio siglo de libros infantiles y juveniles (1935-1985). Salamanca: Fundación Germán Sánchez Ruipérez/Pirámide.

CHECA GODOY, A. (2002): Historia de la prensa pedagógica en España. Sevilla: Secretariado de Publicaciones de la Universidad de Sevilla.

CHIVELET, M. (2009): La prensa infantil en España. Desde el siglo XVIII hasta nuestros días. Madrid: Fundación SM. 
GASCA, L. (1969): Los cómics en España. Barcelona: Lumen

GÓMEZ MOMPART, J. L. (2002): «Ecosistema comunicativo franquista y construcción simbólica y mental de España», en: GARCÍA GALINDO, J. A.; GUTIÉRREZ LOZANO, J. F.; y SÁNCHEZ ALARCÓN, I. (Eds.), La comunicación social durante el franquismo. Málaga: CEDMA, pp. 597-608.

GUAL BORONAT, O. (2013): Viñetas de posguerra. Los cómics como fuente para el estudio de la historia. Valencia: Publicacions de la Universitat de València.

GUBERN, R. (1981): El lenguaje de los cómics. Barcelona: Península/Edicions 62, 4ạ ed.

HELD, J. (1987): Los niños y la literatura fantástica. Función y poder de lo imaginario. Barcelona: Paidós, 3a ed.

HERRERO SUÁREZ, H. (2007): Un yugo para los Flechas. Educación no formal y adoctrinamiento infantil en Flechas y Pelayos. Lleida: Milenio.

LARA, A. (2002): "Los tebeos del franquismo», en: Viviane, A. (Ed.), Historietas, comics y tebeos españoles. Toulouse: Presses Universitaires du Mirail, pp. 44-75.

LORENTE ARAGÓN, J. C. (2000): Los tebeos que leía Franco en la Guerra Civil (19361939). Madrid: IMPHET.

MARTÍNEZ CUESTA, F. J. y ALFONSO SÁNCHEZ, J.M. (2013): «Tardes de enseñanza y parroquia. El adoctrinamiento de las niñas en la España franquista a través de las revistas "Bazar" y "Tin Tan" (1947-1957)». El Futuro del Pasado. Revista electrónica de historia, no 4, pp. 227-253.

MARTÍN MARTÍNEZ, A. (1978): Historia del cómic español: 1875-1939. Barcelona: Gustavo Gili.

MONTERO GARCIA, F. (1987): "Juventud y política: los movimientos juveniles de inspiración católica en España: 1920-1970». Studia Historica. Historia contemporánea, Vol. 5, no 4, pp. 105-121.

- (2001): “Católicos en el franquismo. La Acción Católica”. XX Siglos, Vol. 12, no 49, pp. 4-5.

- (2005): «Las publicaciones periódicas de Acción Católica durante el Franquismo», en: RUIZ SÁNCHEZ, J.L. (Ed.): Catolicismo y comunicación en la historia contemporánea. Sevilla: Universidad de Sevilla, pp. 31-54.

MURIALDI, P. (1986): La Stampa del regime fascista. Roma-Bari: Laterza.

ORTEGA ANGUIANO, J. A. (1986): Los tebeos en España, de la posguerra a la transición (1940-1977). Córdoba: Ayuntamiento de Córdoba. 
PIZARROSO QUINTERO, A. (1990): Historia de la Propaganda. Notas para el estudio de la propaganda política y de guerra. $1^{\text {a }}$ edición. Madrid: Eudema,

SALVADOR, J. (1942): Actividades escolares. Conmemoraciones patrióticas y religiosas. Valencia: Editorial América.

SAN FERNÁNDEZ, F. (1990): Educación No formal en la España de posguerra. Madrid: Universidad Complutense.

SEVILLANO CALERO, F. (1998): Propaganda y Medios de Comunicación en el Franquismo. Alicante: Publicaciones de la Universidad de Alicante.

VÁZQUEZ, J. M. (1963): La prensa infantil en España. Madrid: Doncel.

VÁZQUEZ DE PARGA, S. (1980): Los cómics del franquismo. Barcelona: Planeta.

VIVER PI-SUNYER, C. (1980): «Aproximació a la ideología del franquisme en l'etapa fundacional del Regim». Papers: revista de Sociología, no 14, pp. 11-47.

WEBER, M. (enero-marzo, 1992): «Para una sociología de la prensa». Revista Española de Investigaciones Sociológicas, no 57, pp. 251-259. 